\title{
What makes targets redundant?
}

\author{
G. ROBERT GRICE and JONATHAN M. REED \\ University of New Mexico, Albuquerque, New Mexico
}

\begin{abstract}
Two letter-classification experiments that investigated target-redundancy effects on reaction time (RT) were conducted. Both experiments were replicated with choice reaction time (CRT) and go/no-go (GNG) procedures. In each experiment, there were two single-target conditions, one with a noise letter and one without. In one experiment, the letter classes were two letters that could be of either case. In the second experiment, each class consisted of two different capital letters. In both experiments, there were two redundant-targets conditions, one with identical targets and one with the different members of a class. In both of the GNG experiments, redundancy gains were obtained comparing the different-targets condition with the no-noise, single-target condition. Redundant stimuli are ones that lead to the same response. Visually different stimuli may be processed in parallel and jointly activate a response. GNG procedures are more sensitive than CRT in the investigation of redundancy effects.
\end{abstract}

When target-redundancy effects are investigated by reaction time (RT) experiments, inconsistent results are obtained with choice reaction time (CRT) and go/no-go (GNG) procedures. Grice and Canham (1990), using the GNG procedure, performed replications of two letteridentification experiments that had previously been conducted with CRT. A significant difference from the previous experiments was obtained. In both experiments, a redundancy gain was obtained when a two-target condition was compared with a single target presented alone. In the CRT experiments, no such gain was obtained. The only gain attributable to redundancy was in the comparison of the redundant-targets condition with single targets accompanied by a noise letter. Such results have also been obtained in other CRT experiments. Using GNG, van der Heijden, Schreuder, Maris, and Neerincx (1984) also found a redundancy gain in comparison with a letter without noise.

Grice and Canham (1990) also found one other difference from the CRT procedure. There was no significant difference in RT to single targets presented alone and those accompanied by a noise letter. This difference, typical of CRT, we have previously attributed to a distraction effect of the noise letter. It was suggested by Grice and Canham that these two differences between the RT procedures were the result of the presence of response competition in CRT. This interpretation is also in accord with suggestions of van der Heijden et al. (1984). Grice and Canham also suggested that these results cast doubt on some of the more promising interpretations of redundancy phenomena obtained from CRT experiments. These interpretations have been primarily in terms of concepts

This research was supported by PHS Grant MH16400 from the National Institute of Mental Health. Requests for reprints should be sent to G. R. Grice, Department of Psychology, University of New Mexico, Albuquerque, NM 87131. from perception and attention (e.g., Eriksen, Goettl, St. James, \& Fournier, 1989; Foumier \& Eriksen, 1990; Grice, Canham, \& Gwynne, 1984). It is difficult to see how such notions can account for the difference in results between the two procedures, which differ only in response requirements. One outcome of the above research is the conclusion that the GNG procedure is likely to be more sensitive in demonstrating a redundancy effect and to obtain it in a less complicated fashion. However, for the present, at least, it may be desirable to present comparisons with CRT as a way of providing more complete information.

The research presented here represents an attempt to investigate the nature of redundancy itself. For example, is redundancy dependent on the physical identity of stimuli? To what extent are physically different stimuli redundant, if they share the same name? And on a rather commonsense basis, are different stimuli redundant if one is expected to respond to them in the same way? Another way of asking these questions is to ask whether redundancy is perceptual in nature, a coding problem, or an associative phenomenon. Intimately related to these questions are the problems of separate activation versus coactivation (Miller, 1982) and parallel versus sequential processing (Mordkoff, Yantis, \& Egeth, 1990).

\section{EXPERIMENT 1}

The first experiment provides a basis for comparing stimulus identity and coding identity as possible determiners of redundancy gain. There are two replications-one with CRT and one with the GNG procedure. The experiment is a letter-classification experiment with two classes. Each class consists of both the upper- and lowercase forms of a letter. Stimuli that are physically identical and stimuli not of the same form but with the same name provide a basis for examining redundancy gains. Each replication includes four experimental conditions: a single target pre- 
sented alone, a single target accompanied by an irrelevant noise letter, two identical targets presented together, and upper- and lowercase forms of the same letter also presented together.

\section{Method}

General procedures and method of stimulus presentation were the same as described by Grice and Canham (1990) and Grice and Gwynne (1987). Capital letters were approximately $.29^{\circ}$ wide and $.35^{\circ}$ high. The lowercase letters used were of the same width and $.25^{\circ}$ high. The two letter positions were separated vertically by $3^{\circ}$, center to center. A plus sign used as a fixation point halfway between the letter positions was on at all times except while letters were displayed. The stimuli were light on a dark background. The two target classes were $\mathbf{A}$ and $\mathbf{a}$ and $\mathbf{E}$ and $\mathbf{e}$. These letters were chosen because the upper- and lowercase forms are not similar in shape. In CRT, responses were made by depressing telegraph keys with the right and left forefingers. The subjects were instructed to respond to $A$ or $\mathbf{a}$ with one hand and to $\mathbf{E}$ or $\mathbf{e}$ with the other. The two possible mappings of the right and left responses on the letters were equally divided between the subjects. In the GNG experiment, half of the subjects were instructed to respond to $\mathbf{A}$ and a but not to $\mathbf{E}$ and e. The other half of the subjects received the reverse instructions.

There were four experimental conditions presented equally often in an irregular order. There were two single-target conditions, one with the target presented alone and the other with the letter $\mathbf{Y}$ in the other position. The $\mathbf{Y}$ was always the same case as the target. The target was equally often in the upper and lower positions. There were also two redundant-target conditions, one with identical targets and the other with the same letter in opposite cases. For the opposite-case condition, the two cases were equally often in the upper and lower positions. Each target was presented equally often in the single-target conditions, and each target class was presented equally often in the redundant-targets conditions. All subjects received the same irregular order of trials.

There were a total of 288 trials. The experiment began with 54 practice trials followed by a brief rest. There were then 234 trials without interruption, the first 10 of these treated as further practice. Data were based on the final 224 trials. There were 4 sec between trials. The exposure duration of the letter stimuli was $200 \mathrm{msec}$. Each trial was preceded by $1 \mathrm{sec}$ by an auditory warning signal. The subjects were instructed to look at the fixation point at this signal and to be prepared to respond. They were instructed to respond as quickly as possible while avoiding errors. Feedback was given for errors in CRT and for false alarms in GNG. RTs were recorded milliseconds.

The subjects were undergraduate women from a course in introductory psychology. They received course credit for participation. There were 32 subjects in each replication.

\section{Results}

The mean RTs for Experiment 1 are presented in Figure 1. For the single-target conditions, the upper means indicated are the means of all trials. The faster means are the means of the faster stimulus positions for each subject. For the CRT experiment, the error rate was .022 . In the GNG experiment, the false alarm rate was .024 and the miss rate was .004 .

The choice data, presented in the left panel of Figure 1, indicate a picture quite typical of similar CRT data. Response to the single target with noise is $49 \mathrm{msec}$ slower than to the single target alone. The difference is significant $[t(31)=9.89, p<.001]$. Neither of the two-target conditions show a significant redundancy gain with respect

A 6 × 8 E

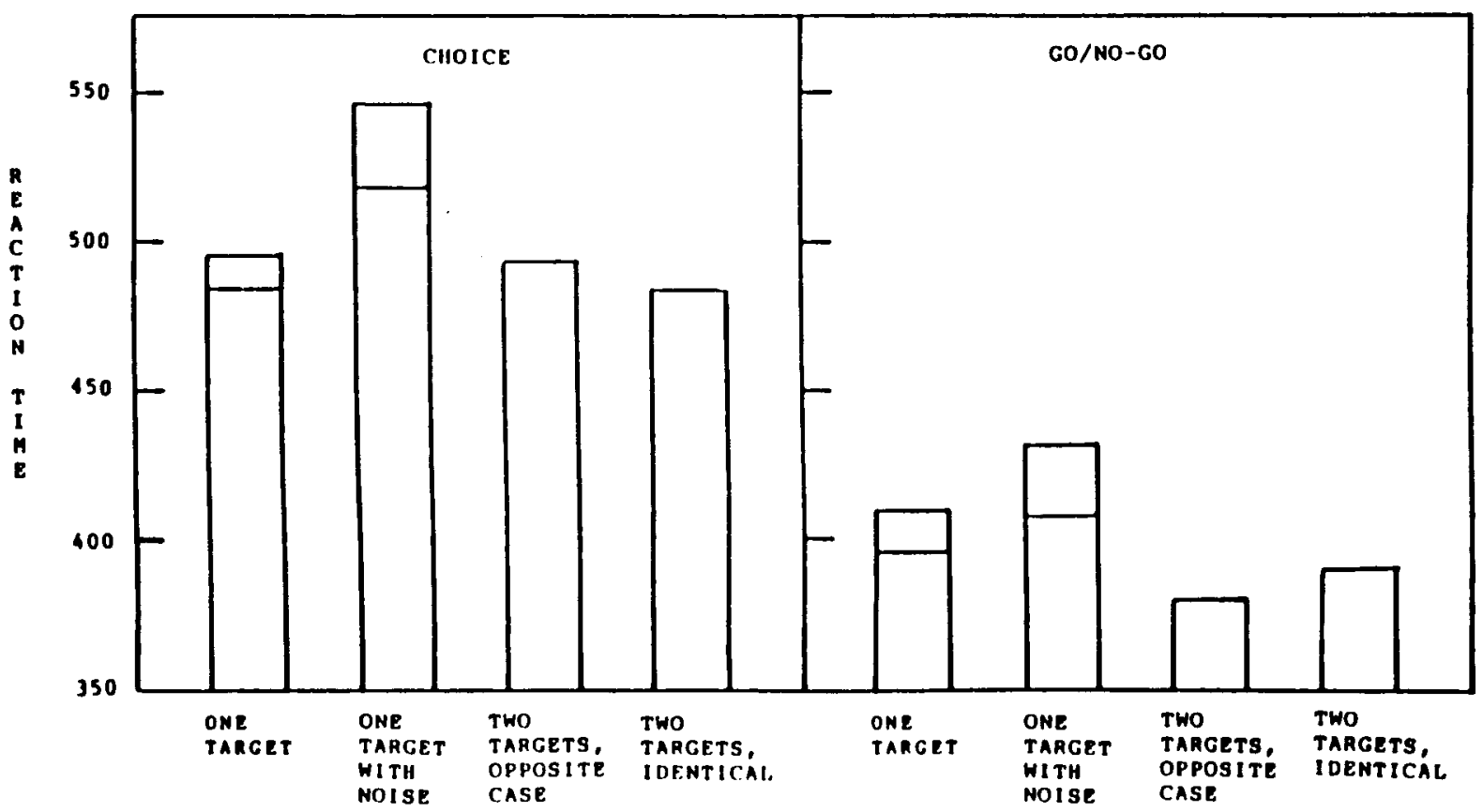

Figure 1. Mean reaction times in milliseconds for each condition in Experiment 1. The two values indicated for the single-target conditions are the means of all trials and the means of the faster positions for each subject. 
to the single target alone. However, both of these conditions are significantly faster than the mean of the faster position for the individual subjects for the single target with noise. The differences are $25 \mathrm{msec}$ for opposite-case targets $[t(31)=3.74, p<.001]$ and $33 \mathrm{msec}$ for identical targets $[t(31)=6.50, p<.001]$. Response to oppositecase targets is $9 \mathrm{msec}$ slower than to identical targets [ $t(31)$ $=2.91, p<.01]$.

The GNG data, presented in the right panel of Figure 1, present a considerably different picture. Of course, the overall faster RTs are to be expected. Possibly the most initially surprising feature is the fact that response to opposite-case redundant targets is faster than to identical targets. This difference of $10 \mathrm{msec}$ is significant $[t(31)$ $=3.00, p<.01]$. This difference is also significantly different from the corresponding difference in the opposite direction for the choice data $[F(1,62)=17.45, p<$ $.001]$.

As in the Grice and Canham (1990) experiment, a redundancy gain was demonstrated in the GNG experiment, for the comparison with the no-noise, single-target condition. The opposite-case, two-target condition was significantly faster by $15 \mathrm{msec}$ than was the mean of subjects' faster positions in the no-noise condition $[t(31)=$ $3.67, p<.001]$. The identical-targets condition was $6 \mathrm{msec}$ faster than the faster positions for the no-noise condition, but this did not reach statistical significance. However, this condition was $19 \mathrm{msec}$ faster than the mean of all trials for the single-target condition, and this difference was significant $[t(31)=3.11, p<.01]$. Both redundant-targets conditions were significantly faster than the single-target condition with noise. ${ }^{1}$

There is one difference here from the Grice and Canham (1990) data. They found no significant difference between the single-target conditions with and without noise. Here, however, the no-noise condition is 21 msec faster than the noise condition, and this difference is significant $[t(31)$ $=4.62, p<.001]$. The results do agree with those of Grice and Canham in that the noise effect is considerably less with GNG than with CRT. The difference between the two differences is significant $[F(1,62)=17.83, p<$ $.001]$.

\section{Tests for Coactivation}

Miller (1982) proposed a way of distinguishing between two alternative classes of models to account for redundancy effects. These two models are (1) coactivation and (2) separate activation, or race models. Coactivation implies that the redundant stimuli each contribute to the activation of a response. There are two kinds of race models. In one of these, the redundant stimuli are processed in parallel, and when the first one reaches criterion, the response occurs (e.g., van der Heijden et al., 1984). The other kind are serial models in which the stimulus with the fastest processing time wins. In such models, if the decision-time distributions of the different channels overlap, RTs will be faster with more than one stimulus present, on purely statistical grounds. Miller showed that for race models, the probability of response to redundant stimuli can never be greater than the sum of the probabilities of response to the separate stimuli at the same time in the cumulative distribution functions (CDFs). Miller called this principle "the race-model inequality." He suggested that it could be evaluated by computing the CDFs for each subject for the redundant-targets condition and for the sum of the single-target stimulus positions. If the redundant condition reaches any quantile significantly sooner than the sum of the single-target positions, the inequality is violated. This leads to the rejection of the entire class of separate activation models and provides evidence for coactivation. Mordkoff et al. (1990) suggest that since the class of serial models is rejected, parallel processing is implied.

To conduct these tests, we computed the quantiles at .1 intervals for the sum of the two stimulus positions for each single-target condition, and for the two redundanttargets conditions in each of the two replications. For the CRT experiment, there were no significant violations of the race-model inequality. There were violations in the GNG experiment. For the opposite-case, redundant-targets condition, the mean of the .1 quantiles preceded the mean of the no-noise quantiles by $7 \mathrm{msec}$, and this difference is significant $[t(31)=2.55, p<.01$, one-tailed test $]$. The mean of the identical-targets .1 quantiles preceded that for the no-noise condition by $5 \mathrm{msec}$, but this difference did not reach significance. It has been the usual practice to test the significance of the difference between quantile means using the $t$ test. However, it has been our experience that it may be preferable to examine the quantile medians and to test by means of the sign test. The reason for this is that there tends to be a rather consistent pattern of violations of the inequality across subjects, while the error term of the $t$ test is inflated by outliers. At the .1 quantile, the opposite-case median preceded that for the no-noise condition by $14 \mathrm{msec}$, and the violation was present for 24 of the 32 subjects $(p=.004)$. At the .2 quantile, the violation of the inequality, indicated by the medians, was $11 \mathrm{msec}$ and was shown by 23 subjects $(p=.01)$. The result of this experiment indicates not only a significant redundancy gain, but coactivation and parallel processing of stimuli that share the same name but are physically different.

\section{EXPERIMENT 2}

By itself, Experiment 1 is only a partial investigation of a coding interpretation of redundancy gain. It is possible that the gain obtained with the opposite-case letters was not dependent on their having the same name but was because they were associated with the same response. Experiment 2 is an investigation of this possibility. The experiment was also a letter-classification experiment with two classes. However, in this experiment, each class consisted of two different letters, both uppercase. Thus, the members of a class differed physically, as in Experiment 1 , but did not have the same name. The letters of 
one class were $\mathbf{A}$ and $\mathbf{D}$, and $\mathbf{E}$ and $\mathbf{R}$ formed the other class. As in Experiment 1, both CRT and GNG procedures were included as two replications.

\section{Method}

The procedures in this experiment were identical to those of Experiment 1 except for the letters used as stimuli and a decreased stimulus exposure of $150 \mathrm{msec}$. One of the redundant-targets conditions consisted of two identical letters, and the other redundant condition was two different letters of the same class. In the CRT experiment, the subjects responded to $A$ and $D$ with one hand and $\mathbf{E}$ and $\mathbf{R}$ with the other, equal numbers of subjects having the two mappings of right and left. In the GNG experiment, half of the subjects responded to $\mathbf{A}$ and $\mathbf{D}$ and half to $\mathbf{E}$ and $\mathbf{R}$. There were 28 subjects from the same population as above in each of the two experiments.

\section{Results}

The means of the experimental conditions are presented in Figure 2. As for Experiment 1, the means of all trials and the means of the faster position for each subject are indicated for the single-target conditions. The error rate for the CRT experiment was .036. For the GNG experiment, the false alarm rate was .035 and the miss rate was.007.

With one exception, the choice experiment replicates the results of Experiment 1 . The exception is that the redundant condition with different letters is $9 \mathrm{msec}$ faster than the identical-targets condition. However, this difference is not significant $[t(27)=1.23$, n.s.]. Since this result conflicts with a significant difference in the opposite direction obtained by Fournier and Eriksen (1990), we suspect sampling error. The single-target condition with noise is $63 \mathrm{msec}$ slower than the no-noise condition $[t(27)=8.91, p<.001]$. Neither of the two-targets conditions was faster than the single-target condition without noise. Typically, both of these conditions showed redundancy gains with respect to the subjects' faster position in the single-target condition with noise. The condition with different letters was 36 msec faster than the single-target condition $[t(27)=5.40, p<.0011$. The identical-letters condition was $27 \mathrm{msec}$ faster than this condition $[t(27)=3.65, p<.01]$.

The pattern of data in the GNG experiment is close to an exact replication of Experiment 1. Again, the singletarget condition with noise was significantly slower than the condition without noise. The difference was $31 \mathrm{msec}$ $[t(27)=5.85, p<.001]$. Also as in Experiment 1, the difference due to noise was significantly smaller than in the choice experiment $[F(1,54)=9.11, p<.005]$.

Another surprising similarity to Experiment 1 was the finding that the condition with different letters as redundant targets was faster than the condition with identical targets. This 9-msec difference was significant $[t(27)=$ $2.55, p<.025]$. The different-letters condition was also $11 \mathrm{msec}$ faster than the mean of the subjects' faster positions in the no-noise, single-target position. This redundancy gain was significant $[t(27)=2.20, p<.05]$. The identical-letters condition was only $2 \mathrm{msec}$ faster than the

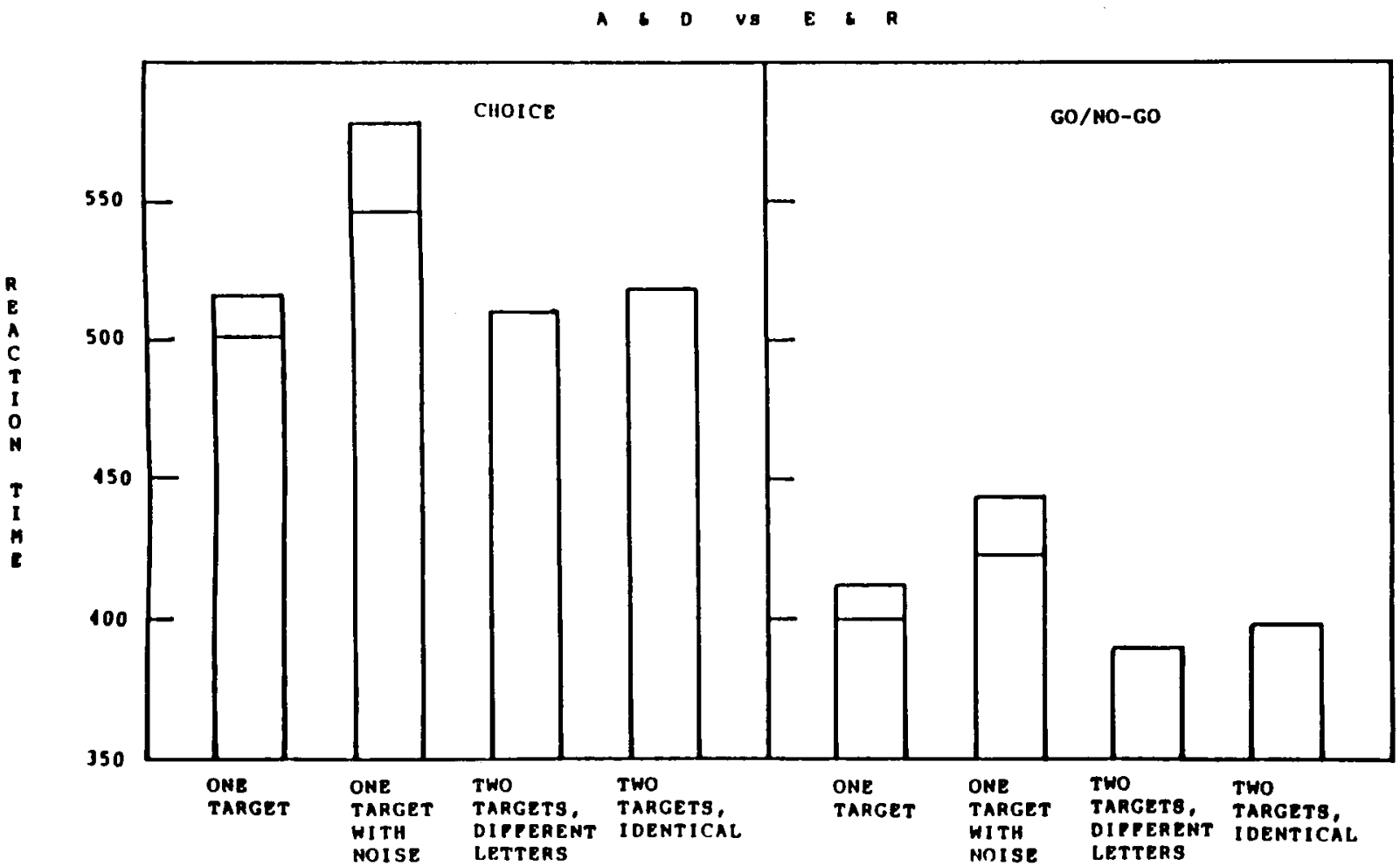

Figure 2. Mean reaction times in milliseconds for each condition in Experiment 2. The two values indicated for the single-target conditions are the means of all trials and the means of the faster positions for each subject. 
mean of the faster positions, but it was $14 \mathrm{msec}$ faster than the mean of all trials in the no-noise condition $[t(27)$ $=2.99, p<.011$. Both of the redundant-targets conditions showed significant gains in comparison with the single targets with noise. ${ }^{2}$

\section{Tests for Coactivation}

To test for violations of the race-model inequality, quantiles for individual subjects were again computed at .1 intervals. The chief interest here is the comparison of the two-targets condition with different letters and the singletarget condition with no noise. The inequality was violated at the earliest three quantiles. At the .1 quantile, the median for the different-letters condition preceded that for the sum of the two positions in the no-noise condition by $15.5 \mathrm{msec}$. The violation was shown by 19 of the 28 subjects, with one tie $(p=.026)$. In terms of the medians, the violations were $26.5 \mathrm{msec}$ for the .2 quantile and $21 \mathrm{msec}$ for the .3 quantile. At each of these quantiles, 20 of the 28 subjects violated the inequality $(p=.018)$. For the identical-targets condition, there were no statistically significant violations of the inequality.

In this experiment, significant redundancy gains were demonstrated by targets that were different letters. Furthermore, violations of the race-model inequality indicate that the different letters were processed in parallel and jointly produced activation of the response. It is the implication of these data that the effects produced by the oppositecase letters in Experiment 1 did not depend on their having the same name.

\section{DISCUSSION}

The use of the GNG procedure in the present research made it possible to obtain important and interesting information about the nature of target redundancy. This information could not be obtained with the choice procedure. What this information indicates is that stimulus redundancy is primarily an associative rather than a perceptual process. Stimuli are redundant if they lead to the same response. We suggest that this is really a commonsense view that would not be surprising to a learning theorist or to a layperson. However, the research is not without perceptual implications. Probably the data are most supportive of a continuous-flow conception (Eriksen \& Schultz, 1979). During the period of continuous growth of associative strength (Grice, 1972), two different stimuli are processed in parallel, both contributing to the growth. The research supports the conclusion of Grice and Canham (1990) that letters separated by $3^{\circ}$ of visual angle may be processed in parallel. The additional information is added that these stimuli need not be visually identical. Fournier and Eriksen (1990) suggested that coactivation is a perceptual process dependent on visual identity. These data indicate that this is not necessarily the case.
There seem to be no essentially new conclusions to be drawn from the CRT experiments. The main result of their inclusion in this research seems to be the illustration that the GNG procedure is more sensitive in the investigation of stimulus redundancy. We agree with Grice and Canham (1990) that the main source of difference between the two procedures is the presence of response competition in CRT.

The finding in both experiments that nonidentical redundant stimuli were more effective than identical stimuli was not an expected result. We suggest that two different redundant stimuli may provide additional associative information compared with two stimuli that are the same. Following an oral presentation of these data, George Sperling (personal communication, November 1990) suggested that the result could be due to the availability of feature detectors. For two visually different stimuli, a larger population of such detectors would be available. To the authors, this seems a bit speculative but appears plausible.

Both of the present experiments differed from the Grice and Canham (1990) data concerning their finding of no significant differences between single-stimulus conditions with and without noise. It is shown here that a distraction effect of noise can be obtained with the GNG procedure. However, in both experiments, the effect was significantly smaller than in the CRT experiments. The conclusion now seems to be that GNG reduces distraction effects in comparison with CRT. The reason for this difference from the Grice and Canham data is not known. However, the main difference between their experiments and the present ones is that here the response was made to either of two different stimuli, while there was only one stimulus in the Grice and Canham experiment. Conceivably this could affect the role of distracting stimuli.

In summary, the major conclusion of this research is that an associative interpretation of redundancy is supported rather than interpretations in terms of stimulus identity or stimulus coding. In both GNG experiments, maximum redundancy gains were obtained with nonidentical targets. This was true whether or not the targets had the same name. The answer to the question raised in the title is implied by these results. Targets are redundant if they are associatively related to a common response.

\section{REFERENCES}

Eriksen, C. W., Goettl, B., St. James, J. D., \&ournier, L. R. (1989). Processing redundant signals: Coactivation, divided attention, or what? Perception \& Psychophysics, 45, 356-370.

ErIKsen, C. W., SChultz, D. W. (1979). Information processing in visual search: A continuous flow conception and experimental results. Perception \& Psychophysics, 25, 249-263.

Fournier, L. R., ERIKSEN, C. W. (1990). Coactivation in the perception of redundant targets. Joumal of Experimental Psychology: Human Perception \& Performance, 16, 538-550.

Grice, G. R. (1972). Conditioning and a decision theory of response evocation. In G. H. Bower (Ed.), The psychology of leaming and motivation (Vol. 5). New York: Academic Press. 
Grice, G. R., \& CANHAM, L. (1990). Redundancy phenomena are affected by response requirements. Perception \& Psychophysics, 48, 209-213.

Grice, G. R., Canham, L., Gwynne, J. W. (1984). Absence of a redundant-signals effect in a reaction time task with divided attention. Perception \& Psychophysics, 36, 565-570.

GrJCE, G. R., \&WYNNE, J. W. (1987). Dependence of target redundancy effects on noise conditions and number of targets. Perception \& Psychophysics, 42, 29-36.

Miller, J. (1982). Divided attention: Evidence for coactivation with redundant signals. Cognitive Psychology, 14, 247-279.

MoRDKoFf, J. T., YANTIS, S., EGETH, H. E. (1990). Detecting conjunctions of color and form in parallel. Perception \& Psychophysics, 48, 157-168.

van der Heuden, A. H. C., Schreuder, R., Maris, L., a Neerincx, M. (1984). Some evidence for correlated separate activation in a simple letter-detection task. Perception \& Psychophysics, 36, 577-585.

\section{NOTES}

1. There is one aspect of the data that suggests a possible complication in the interpretation of this experiment. The uppercase letters were larger and apparently more distinctive than the lowercase letters. The result was faster RTs for the uppercase letters. Averaged over the three conditions with separate presentations of the two targets, RTs in the GNG experiment were $35 \mathrm{msec}$ faster for the capital letters. In the CRT experiment, this advantage was $\mathbf{4 4} \mathrm{msec}$. Both differences are statistically reliable. The possible complication is that the advantage of the opposite-case, redundant-targets condition in the GNG experiment was due to the presence of a more distinctive capital letter on every trial. The data, however, do not support this interpretation. The most obvious problem is that the differences between the opposite-case and identical-targets conditions were in opposite directions in the CRT and GNG experiments. As indicated above, this interaction was highly significant. The displays in the two experiments were identical, and the uppercase advantage applied to both. It is obvious that the suggested interpretation cannot account for this pattern of data.

A further examination of the GNG data indicated that the redundancy effect of the opposite-case condition was $16 \mathrm{msec}$ faster than the mean of the uppercase trials for the single-target condition. The difference is significant $[t(31)=4.14, p<.001]$. This means that the lowercase target contributed significantly to the redundancy effect, even in comparison with the more distinctive uppercase single target.

2. In Experiment 2, there is no evidence for a consistently more distinctive letter that could account for the superior redundancy effect of the different-letters condition in the GNG experiment. For the subjects responding to $\mathbf{A}$ and $\mathbf{D}$ in this condition, RTs to $\mathbf{D}$ averaged $7.2 \mathrm{msec}$ faster than to $\mathbf{A}$. For subjects responding to $\mathbf{E}$ and $\mathbf{R}$, the RTs to $\mathbf{E}$ averaged $1.6 \mathrm{msec}$ faster than to $\mathbf{R}$. Neither of these differences approached statistical significance. However, in spite of this evidence, we have conducted an analysis parallel to the one reported for Experiment 1 in Note 1. The differences between the letters for the no-noise condition were in the same direction, but larger, than the overall averages, that is, $15 \mathrm{msec}$ for $\mathbf{A}$ and $\mathbf{D}$ and $11 \mathrm{msec}$ for $\mathbf{E}$ and $\mathbf{R}$. Thus, we combined the trials on which $\mathbf{D}$ and $\mathbf{E}$ were presented. The mean of the differentletters, redundant-targets condition was $16.3 \mathrm{msec}$ faster than the mean of these trials for the no-noise condition. This difference is statistically significant $[t(27)=2.822, p<.01]$. It is our conclusion that the hypothesis that the superiority of rectundant-targets conditions with nonidentical stimuli is the result of the presence of a distinctive target is not supported by the data of either experiment.

(Manuscript received September 13, 1991; revision accepted for publication December $9,1991$. 